\title{
Toward Live Longer, Younger, Happier and Healthier: Reprogramming for a New Start or Just a Turn?
}

\section{Yue Zhang*}

Research Center, CHUM, Notre Dame Hospital, Montreal, Canada

Whether we could live longer, younger, happier, and healthier as well as especially cancer-free in the future? Reprogramming in stem cell therapy and regenerative medicine could shed light on it [1]. Whether the DNA of the mature cell still hold all the information needed to develop all cells in the organism? 50 years ago, most if not all biologists could have said "no." However, Sir Gurdon applied his frog nuclear tranplantation (cloning) [2] to say "yes", it thus is feasible with one reprogramming for a new start i.e. the specialisation of cells is reprogrammable. At that time, he even predicted that the mammalian animal cloning would come up during following 50 years. In fact, one of most fascinating events is the Dolly cloning around the turn of this century. So his finding proved that cells all carry the same genes, with other factors telling the cell which to use. What are other factors? Now we know that epigenetic factors are among them. Though not as stable as genome, epigenome holds genetic information and its dynamics reflects its communication with the environment. Particularly, DNA methylation and histone modification, two major epigenetic modifications, play significant effects on stem cell and tumorigenesis. With their excellent expertise, Dr. Yang et al. [3] discussed on epigenome in stem cell and tumorigenesis alongside next generation sequencing technologies, which have directly contributed to the recent advances in epigenome research of stem cell and cancer. Amazingly, another reprogramming for a new start, induced pluripotent stem cell (iPS) technology, has been invented by Takahashi and Yamanaka [4], which has been spotlighted as 2012 Nobel Prize in medicine and physiology together with Gurdon's work. It is awesome that several articles in this special issue have placed emphasis on this direction. Myriad information for its significance, the advancements, and perspectives has been well described. Do not be lost in my bold presumption, but let us to make a broad definition of tumorigenic cell, which includes iPS cell as one exceptional type just as we warm welcome it. On another hand, cancer stem cells (CSCs), also like stem cell, with potentials of self-renewal and differentiation into terminal cells, are the subpopulation of cells within a tumor proposed to be responsible for tumor initiation, relapses, and resistance to chemotherapeutic drugs. CSCs could be initiated with mal-functional master regulators including those identified to switch the multicellularity [5] or Warburg effect [6,7]. Therefore everything here could be possibly unified under cellular reprogramming. In reality, iPSc indeed own the potentials of tumorigenicity [4]. Gao et al. [8] started their review from the establishment of the first iPS cell line, then continued to cell therapy, human disease modeling, and drug discovery. Further they pointed out that the use of peripheral blood facilitates research on iPS cells and enables the establishment of patient-specific iPS cells and consider that clinical therapy based on iPS cells will rapidly develop. Indeed, Dr. Yamanaka did mention that iPS -based clinical trial will be performed in Japan in two years (personal communication).

Clearly, cellular reprogramming is not only for a new start to stem cells but also more frequently for a switch in critical turn points, such as recently-resurgent direct conversion between differentiated cells or therapeutic reprogramming. It has been previously hypothesized that reprogramming from the critical turning points spontaneously happens often in aging-related complex diseases (ACDs), such as cancer [5,9], neurodegenerative diseases [10], or osteoarthritis [11]. Thus this special issue also includes one study related to Alzheimer's Disease [12]. In general, ACDs may be alongside the decrease of dilution of cellular damage and the robustness of mechanisms in organisms may protect a system failure to some cancers until adulthood $[9,13]$. Cell division and proliferation provide some dilutions of cellular damage. However, cancer requires indefinite proliferation potential. On the other hand, conventional theories for aging and carcinogenesis are facing some challenges, which could lead to potential paradigm shift. The genetic regulatory network (GRN)-based cell attractors theory emerged to contribute to our better understanding of ACDs $[1,8,14]$, but far from completed. Most importantly, human artificial intervention with chemicals and/or transcriptional factors could turn abnormal reprogramming back to normal-like. In this issue, focus on CSC-targeted therapy, Dr. Chen and his colleagues [14] observed that the percentage of CSC in IMCE-Ras cells significantly decreased after a short treatment with DNA methylation inhibitor 5-aza-2'deoxycytidine (5-Aza-dC), indicating that DNA methylation may be critical for self-renewal and maintenance of CSCs. Additionally, double knockout of DNA methyltransferase 1 and DNA methyltransferase 3b resulted in loss of $>95 \%$ DNA methylation in colon cancer cell line, and this systemic epigenetic reprogramming causes complete loss of tumorigenicity. Thus a combination of classical chemotherapeutic drugs and global reprogramming of DNA methylation may be an effective treatment of colon cancer and even possibly with low dosage [15].

May be inspired and further encouraged by iPS, the direct conversion [16], particularly with transcriptional factors, is one unique cellular reprogramming in animals bypass a new start. Rare genes are thus far identified as master regulator for epigenetic reprogramming at some critical turn points, including SIRT6 [6], SIRT1 [17], TET2 [7] and aforementioned $\mathrm{Mi}-2 \beta$ [5], whose deficiency is enough to transform normal cells into diseased even tumor cells because they may control several aspects of stem cell, ageing and cancer biology. Besides, the components, which highly orchetsrate cell cycle and cell division, function in concerted with Mi-2 $\beta$ at a system level (unpublished). Any further other factors? Interestingly, one latest study from Dr. John Dick group [18] says that factors beyond DNA sequence and epigenetic

*Corresponding author: Yue Zhang, Research Center, CHUM, Notre Dame Hospital, Montreal,1560 Sherbrooke Street East, Pavillon DeSève, Montreal, Quebec, Canada, E-mail: zy1001@yahoo.com

Received December 24, 2012; Accepted December 27, 2012; Published December 28, 2012

Citation: Zhang Y (2012) Toward Live Longer, Younger, Happier and Healthier: Reprogramming for a New Start or Just a Turn? Human Genet Embryol S2:e001. doi:10.4172/2161-0436.S2-e001

Copyright: (c) 2012 Zhang Y. This is an open-access article distributed under the terms of the Creative Commons Attribution License, which permits unrestricted use, distribution, and reproduction in any medium, provided the original author and source are credited. 
Citation: Zhang Y (2012) Toward Live Longer, Younger, Happier and Healthier: Reprogramming for a New Start or Just a Turn? Human Genet Embryol S2:e001. doi:10.4172/2161-0436.S2-e001

Page 2 of 2

chemical groups attached to it, which influences gene expression, or even environmental influences -shape tumor-cell conduct. So far, the cellular reprogramming is inviting but not meets for all aims. Embryonic stem (ES) cells are still used in various fields for diverse purposes, including gene targeting, cell therapy, tissue repair, organ regeneration, and so on. Dr. Burlacu [19] discussed on Umbilical Cord Blood (UCB) as new sources of stem cells with significant clinical benefits in the regenerative medicine and as a valuable alternative source of haematopoietic stem cells for the treatment of various disorders. In addition, in this special issue, Dr. Xiao [20] placed their emphasis on epigenetic regulation of DNA methylation, histone modifications, non-coding microRNA regulation and chromatin remodelling, which are essential for the maintenance and differentiation of hematopoietic stem/progenitor cells.

However, the evolution will eventually come to ethical disputes which we could bet on, then it could encourage stem cell therapy even human cloning just as Sir Gurdon predicted to show up during next 50 years. Yes or no, we will see. Finally, from system biology down to individual mutation and molecular level, many questions remain: how to find out such critical turning points, why some abnormal dramatic systematic genetic reprogramming take place at such critical turning points during the development and adulthood, what are in charge of such switch, whether and how we could intervene them toward expected desirable directions by means of pharmacological epigenetic reprogramming and/or metabolic reprogramming, etc.

\section{References}

1. Huang S, Ernberg I, Kauffman S (2009) Cancer attractors: a systems view of tumors from a gene network dynamics and developmental perspective. Semin Cell Dev Biol 20: 869-876.

2. Gurdon JB (1962) Adult frogs derived from the nuclei of single somatic cells Dev Biol 4: 256-273.

3. Jun Li TW, Xiuge Zhang, Xu Yang (2011) The Contribution of Next Generation Sequencing Technologies to Epigenome Research of Stem Cell and Tumorigenesis. Human Genet Embryol S2-001.

4. Takahashi K, Yamanaka S (2006) Induction of pluripotent stem cells from mouse embryonic and adult fibroblast cultures by defined factors. Cell 126 663-676.

5. Zhang Y, Moriguchi H (2011) Chromatin remodeling system, cancer stem-like attractors, and cellular reprogramming. Cell Mol Life Sci 68: 3557-3571.
6. Sebastián C, Zwaans BM, Silberman DM, Gymrek M, Goren A, et al. (2012) The Histone Deacetylase SIRT6 Is a Tumor Suppressor that Controls Cancer Metabolism. Cell 151: 1185-1199.

7. Quivoron C, Couronné L, Della Valle V, Lopez CK, Plo I, et al. (2011) TET2 inactivation results in pleiotropic hematopoietic abnormalities in mouse and is a recurrent event during human lymphomagenesis. Cancer Cell 20: 2538.

8. Jiang W, Zhang J, Yang X, Gao J (2011) Induced Pluripotent Stem Cells: Origins and Directions. Human Genet Embryol (S2: 002)

9. Zhang Y (2011) New Concepts of Germline Gene -reactivated Cancer. Human Genet Embryol 1: e101.

10. Zhang $Y$ (2012) Ageing and cancer: breaking the "Don't put all eggs in one basket" and natural "self-organisation", and their potential reprogramming via modulation of Mi-2/NuRD and mTOR kinase ( in press). Enzyme engineering.

11. Zhang Y (2012) New Frontiers of Aging Reversal and Aging-Related Diseases Reprogramming. Med Advancements in Genet Engg 1: e101.

12. Yun W, Xu S, Bi J (2012) The Association between the MTR Gene A2576G Polymorphism and Alzheimer's Disease: a Meta Analysis Study. Human Genet Embryol S2: 003

13. Zhang Y, Calderwood SK, Moriguchi H (2012) Visualization of Archaic Cancer Attractors and Chromatin Regulators Connecting to Ageing, Robustness and Cellular Reprogramming. Nova Science Publishers, New York.

14. Wang S, Lo PK, Chandrashekaran V, Duan X, Berger FG, et al. (2012) Enrichment and Selective Targeting of Cancer Stem Cells in Colorectal Cance Cell Lines. Human Genet Embryol S2: 006.

15. Tsai HC, Li H, Van Neste L, Cai Y, Robert C, et al. (2012) Transient low doses of DNA-demethylating agents exert durable antitumor effects on hematological and epithelial tumor cells. Cancer Cell 21: 430-446.

16. Nizzardo M, Simone C, Falcone M, Riboldi G, Comi GP, et al. (2012) Direct reprogramming of adult somatic cells into other lineages: past evidence and future perspectives. Cell Transplant.

17. Calvanese V, Fraga MF (2011) SirT1 brings stemness closer to cancer and aging. Aging (Albany NY) 3: 162-167.

18. Kreso A, O’Brien CA, van Galen P, Gan O, Notta F, et al. (2012) Variable Clona Repopulation Dynamics Influence Chemotherapy Response in Colorectal Cancer. Science.

19. Burlacu A (2012) Umbilical Cord Blood: Medical Waste or Important Source of Stem Cells? Human Genet Embryol S2: 005.

20. Wang Y, Moore BT, Peng X, Xiao P (2012) Epigenetics and Hematopoietic Stem or Progenitor Cells. Human Genet Embryol S2: 004.

This article was originally published in a special issue, Epigenetics, stem cells and tumorigenicity handled by Editor(s). Dr. Yue Zhang, Harvard Medical School, USA; Yujing Li, Emory University School of Medicine, USA Yanhong Ji, Xian Jiaotong University, China 